\title{
A covariant determination of the Weyl canonical frames in Petrov type I spacetimes
}

\author{
Joan Josep Ferrando $†$ and Juan Antonio Sáez $\ddagger$ \\ $\dagger$ Departament d'Astronomia i Astrofísica, Universitat de València, E-46100 Burjassot, València, \\ Spain \\ $\ddagger$ Departament d’Economia Financera i Matemàtica, Universitat de València, E-46010 València, \\ Spain
}

Received 1 April 1996, in final form 24 September 1996

\begin{abstract}
A covariant algorithm is given to obtain principal 2-forms, Debever null directions and canonical frames associated with Petrov type I Weyl tensors. The relationship between these Weyl elements is explained, and their explicit expressions depending on Weyl invariants are obtained. These results are used to determine a cosmological observer in type I universes, and their usefulness in spacetime intrinsic characterization is shown.
\end{abstract}

PACS numbers: $0240 \mathrm{H}, 0420 \mathrm{C}$

\section{Introduction}

Algebraic classification of the Weyl tensor has been considered from two different points of view. The approach initiated by Petrov [1] studies the eigenvalue and eigenvector problem for the Weyl tensor regarded as an endomorphism on the 2-form space. This classification was completed by Géhéniau [2] and Bel [3] considering, not only the number of independent invariant subspaces, but also the eigenvalue multiplicity. In this framework we find in a natural way the notion of principal 2-form which was analysed widely by Bel [4] for the different algebraic types. An alternative viewpoint $[5,6]$ consists in the study of the relative positions between the 'light cones' determined on the 2-form space by the canonical metric and the Weyl tensor as a quadratic form. From this angle, which is equivalent to the spinorial approach [7], the Weyl classification implies studying the roots of a fourth degree algebraic equation with complex coefficients. A Debever null direction of the Weyl tensor corresponds to every root of this equation $[8,9,7]$.

Both approaches show a richness and variety of directions and 2-planes associated with the Weyl tensor. These geometric elements are generically different from either viewpoint, but the more degenerate the Weyl tensor is, the more common these elements become. Indeed, the relation between the Debever null directions and the principal 2-forms is complex, and only the multiple Debever directions are fundamental directions of null principal 2-forms [4]. In the most regular case, Petrov type I, a Debever direction never coincides with a principal direction of a principal 2-form, and their relation depends strongly on the four Weyl scalar invariants.

$\S$ E-mail: joan.ferrando@uv.es

0264-9381/97/010129+10\$19.50 (C) 1997 IOP Publishing Ltd 
Following the work of Sachs [10], where he gave the hierarchy of equations for the multiplicity of the Debever directions, and the publication of the d'Inverno and RussellClark [11] algorithm, the null direction approach became popular; since then the geometry of principal 2-forms has seldom been considered in the literature. Therefore, although the geometric richness of both points of view were underlined and widely studied in pioneering papers by Penrose [7], Bel [4] and Debever [6] some years ago, until now the relation between them has not been sufficiently analysed for the type I case. However, when considering this subject we can quote the papers by Trümper [12] and Narain [13], or those more recent ones by McIntosh et al [14] and Bonanos [15]. In the latter, a method is given to determine Weyl canonical frames from null Debever directions. In the other three works, we can find expressions for Debever directions in terms of an orthonormal canonical frame in several particular cases: purely electric, purely magnetic and when the four Debever directions span a 3-plane.

Here we extend these results about Petrov type I and present a geometric interpretation of them. We show that the Debever directions are the principal directions of two characteristic 2 -forms associated to the Weyl tensor. We give a covariant expression for these 2-forms in terms of principal 2-forms and a complex invariant scalar. Furthermore we also determine principal 2-forms in a covariant way. These results provide an algorithm to determine the Debever directions.

The covariant determination of the Ricci eigenvectors and its causal character [16] is a necessary tool in the characterization of spacetimes obeying their energy content. In particular its usefulness has been shown in building a Rainich theory for the thermodynamic perfect fluid [17]. In a similar way, in addition to knowing its classification and giving algorithms to distinguish every case, a complete algebraic study of the Weyl tensor implies knowing the covariant determination of the Weyl eigenvectors, i.e. of the principal 2-forms. A complete analysis of this subject, considering all Petrov types, has been developed elsewhere $[18,19]$. Here we only present the results for type I and we use them to determine the Weyl canonical frames in an alternative way to the Bonanos method [15]. The aforementioned paper departs from the Debever directions, whereas here we give covariant expressions, in terms only of the Weyl tensor, using principal 2-forms.

The usefulness of Weyl canonical frames in the metric equivalence problem is well known. In the algebraically special cases there is a close relationship between canonical frames and Debever directions, but in the algebraically general one its relation involves Weyl scalar invariants and, as a consequence, canonical frame determination is more difficult. Here, we give a geometric interpretation of this relationship and offer an algorithm which allows us to obtain canonical frames directly without solving any algebraic equation.

The paper is organized as follows. In section 2 we introduce the formalism used in the paper and recapitulate some properties about 2-forms, in particular the covariant way used to determine their principal directions. We also point out the bijection and give its covariant expression between orthonormal frames on the tangent space and orthonormal bases on the self-dual 2-form space.

In section 3 we summarize the characterization and the canonical expressions of a type I Weyl tensor, and present the Weyl concomitants that enable us to obtain the three orthonormal principal 2-forms. From them, and using the results in section 2, we give a covariant algorithm to determine the Weyl canonical frames.

The connection between principal 2-forms and Debever directions is analysed in section 4 . We give a clear interpretation in the 2-form space framework, and comment on the relationship between our approach and the previous results quoted above.

Finally, in section 5 we apply our results to determine a cosmological observer in Petrov 
type I universes. This allows us to give an intrinsic characterization of an interesting family of spacetimes: those that admit an hypersurface orthogonal observer measuring isotropic radiation.

\section{The self-dual 2-forms space}

Let $\left(V_{4}, g\right)$ be the spacetime of signature $\{-,+,+,+\}$. On the six-dimensional vectorial space of the 2-forms, two different metrics can be considered: the usual one induced by the spacetime metric, $G=\frac{1}{2} g \wedge g\left((g \wedge g)_{\alpha \beta \gamma \delta}=2\left(g_{\alpha \gamma} g_{\beta \delta}-g_{\alpha \delta} g_{\beta \gamma}\right)\right)$, and that defined by the metric volume element $\eta$, i.e.

$G(F, H) \equiv(F, H)=\frac{1}{4} G_{\alpha \beta \mu \nu} F^{\alpha \beta} H^{\mu \nu} ; \quad \eta(F, H) \equiv(* F, H)=\frac{1}{4} \eta_{\alpha \beta \mu \nu} F^{\alpha \beta} H^{\mu \nu}$.

The scalar invariants of a 2 -form $F$ are defined by its squares calculated with these two metrics: $(F, F)$ and $(* F, F)$. A 2-form is usually named non-null or regular when $(F, F)^{2}+(* F, F)^{2} \neq 0$, and null or singular otherwise. Simple 2-forms satisfy $(* F, F)=0$. The principal directions $l_{ \pm}$of a regular 2 -form $F$ are the common (null) eigenvectors to $F$ and $* F$ :

$$
l_{ \pm} \wedge F\left(l_{ \pm}\right)=0 ; \quad l_{ \pm} \wedge * F\left(l_{ \pm}\right)=0 .
$$

A unitary 2-form is a simple 2-form $U$ such that $(U, U)=-1$. In terms of its principal directions, we have $U=l_{-} \wedge l_{+}$.

Principal directions of a 2-form may be determined by solving the eigenvector equations (1). However, we can also use a covariant method that enables us to obtain them without solving any equations [20]. In particular, principal directions of a unitary 2-form $U$ are:

$$
l_{ \pm} \propto[U \times U \underline{+} U](x)
$$

where $x$ is an arbitrary timelike direction and $U \times U=\operatorname{tr}_{23} U \otimes U$.

A self-dual 2-form is a complex 2-form $\mathcal{F}$ such that $* \mathcal{F}=\mathrm{i} \mathcal{F}$. We can associate to every real 2-form $F$ the self-dual 2-form $\mathcal{F}=\frac{1}{\sqrt{2}}(F-\mathrm{i} * F)$. The endowed metric on the three-dimensional complex subspace of the self-dual 2 -forms is $\mathcal{G}=\frac{1}{2}(G-\mathrm{i} \eta)$. Then, for every self-dual 2-form, the complex scalar invariant $\mathcal{G}(\mathcal{F}, \mathcal{F}) \equiv(\mathcal{F}, \mathcal{F})=(F, F)-\mathrm{i}(* F, F)$ may be considered. A self-dual 2 -form $\mathcal{H}$ is a null vector for $\mathcal{G}$ when $H$ is singular, and a $\mathcal{G}$-unitary self-dual 2 -form $\mathcal{U}$ corresponds to every real unitary 2-form $U$. Then, equations (1) for the principal directions of $U$ can be written as:

$$
l_{\underline{ \pm}} \wedge \mathcal{U}\left(l_{\underline{+}}\right)=0
$$

Let $\left\{e_{\alpha}\right\}_{\alpha=0}^{3}$ be an oriented and orthochronous orthonormal frame. Then, if we define $U_{i}=e_{0} \wedge e_{i}$, we obtain a self-dual orthonormal frame $\left\{\mathcal{U}_{i}\right\}_{i=1}^{3}$, which has an induced orientation given by

$$
\mathcal{U}_{k}=\mathrm{i} \sqrt{2} \epsilon_{i j k} \mathcal{U}_{i} \times \mathcal{U}_{j}
$$

Conversely, every oriented (by (4)) orthonormal frame $\left\{\mathcal{U}_{i}\right\}$ on the self-dual 2-forms space has a unique oriented and orthochronous orthonormal frame $\left\{e_{\alpha}\right\}$ associated with it such that $U_{i}=e_{0} \wedge e_{i}$. This one-to-one map follows from the isomorphism between the proper orthochronous Lorentz group, $\mathcal{L}_{+}^{\uparrow}$, and the group $S O(3, \mathcal{C})$ of the proper orthogonal transformations on the self-dual 2-forms space:

$$
\mathcal{L}_{+}^{\uparrow} \longrightarrow S O(3, \mathcal{C}) ; \quad L \longrightarrow \frac{1}{2}[L \wedge L-\mathrm{i} *(L \wedge L)] .
$$


We know explicitly $\left\{\mathcal{U}_{i}\right\}$ in terms of $\left\{e_{\alpha}\right\}$; we will now determine covariant expressions which give $\left\{e_{\alpha}\right\}$ in terms of $\left\{\mathcal{U}_{i}\right\}$. The associated real 2-form, $U_{i}=\operatorname{Re}\left(\sqrt{2} \mathcal{U}_{i}\right)$, verifies $U_{i} \times U_{i}=-e_{0} \otimes e_{0}+e_{i} \otimes e_{i}$. Then, it is easy to show that

$$
e_{0} \otimes e_{0}=\frac{1}{2}\left(g-\sum_{i=1}^{3} U_{i} \times U_{i}\right) \equiv P_{0} .
$$

Thus, we arrive at the following

Lemma 1. If $\left\{e_{\alpha}\right\}$ is an oriented and orthochronous orthonormal frame and $U_{i}=e_{0} \wedge e_{i}$, then the term $\left\{\mathcal{U}_{i}\right\}, \mathcal{U}_{i}=\frac{1}{\sqrt{2}}\left(U_{i}-\mathrm{i} * U_{i}\right)$, is an oriented frame on the self-dual 2 -forms space. This is a one-to-one map and its inverse is given by:

$$
e_{0}=\frac{-P_{0}(x)}{\sqrt{P_{0}(x, x)}} ; \quad e_{i}=U_{i}\left(e_{0}\right)
$$

with

$$
P_{0} \equiv \frac{1}{2}\left(g-\sum_{i=1}^{3} U_{i} \times U_{i}\right)
$$

and where $x$ is an arbitrary future-pointing vector.

\section{Principal 2-forms and canonical frames}

The algebraic classification of the Weyl tensor $W$ can be obtained [2] by studying the linear map defined by the self-dual Weyl tensor $\mathcal{W}=\frac{1}{2}(W-\mathrm{i} * W)$ on the self-dual 2-form space. In terms of its complex scalar invariants, $a=\operatorname{tr} \mathcal{W}^{2}, b=\operatorname{tr} \mathcal{W}^{3}$, the characteristic equation reads

$$
x^{3}-\frac{1}{2} a x-\frac{1}{3} b=0
$$

and their roots are

$$
\alpha_{k}=\beta \mathrm{e}^{\frac{2 \pi k}{3} \mathrm{i}}+\frac{a}{6 \beta} \mathrm{e}^{-\frac{2 \pi k}{3} \mathrm{i}},
$$

with

$$
\beta=\sqrt[3]{\frac{1}{6}\left(b+\sqrt{b^{2}-\frac{a^{3}}{6}}\right)} .
$$

The Weyl tensor is Petrov type I if (6) has three different roots $\left\{\alpha_{i}\right\}$, which is equivalent to the condition $6 b^{2} \neq a^{3}$. In this case an orthonormal frame $\left\{\mathcal{U}_{i}\right\}$, built up with eigenvectors of $\mathcal{W}$, exists: they are the principal 2-forms of the Weyl tensor [4]. Then, the self-dual Weyl tensor takes the canonical expression

$$
\mathcal{W}=-\sum_{i=1}^{3} \alpha_{i} \mathcal{U}_{i} \otimes \mathcal{U}_{i}
$$

Now we consider the determination of the principal 2-forms $\left\{\mathcal{U}_{i}\right\}$ in terms of the Weyl tensor. From the characteristic equation,

$$
\prod_{i=1}^{3}\left(\mathcal{W}-\alpha_{i} \mathcal{G}\right)=0,
$$


it follows that $\left(\mathcal{W}-\alpha_{i} \mathcal{G}\right)\left(\mathcal{P}_{i}(\mathcal{X})\right)=0$ for every self-dual 2-form $\mathcal{X}$, where

$$
\mathcal{P}_{i}=\prod_{j \neq i}\left(\mathcal{W}-\alpha_{j} \mathcal{G}\right)
$$

Thus $\mathcal{P}_{i}(\mathcal{X})$ belongs to the eigenspace corresponding to the eigenvalue $\alpha_{i}$, that is to say, $\mathcal{P}_{i}$ is the projection map on this eigenspace. In consequence, we are lead to the following

Proposition 1. Let $\mathcal{W}$ be the self-dual Weyl tensor of a Petrov type I spacetime. The principal 2-form $\mathcal{U}_{i}$ corresponding to the eigenvalue $\alpha_{i}$ may be obtained as

$$
\mathcal{U}_{i}=\frac{\mathcal{P}_{i}(\mathcal{X})}{\sqrt{-\mathcal{P}_{i}^{2}(\mathcal{X}, \mathcal{X})}}
$$

with $\mathcal{P}_{i}=\mathcal{W}^{2}+\alpha_{i} \mathcal{W}+\left(\alpha_{i}^{2}-\frac{1}{2} a\right) \mathcal{G}$, and where $\mathcal{X}$ is an arbitrary self-dual 2-form such that $\mathcal{P}_{i}(\mathcal{X}) \neq 0$.

This proposition provides a covariant algorithm to obtain the principal 2-forms of a type I Weyl tensor. They are given by (8) and are determined up to sign and permutation. Thus, we can consider 24 oriented eigenframes $\left\{\mathcal{U}_{i}\right\}$ : for every permutation, the sign of two of them gives us four possibilities, the third being given by (4).

The one-to-one map defined in lemma 1 associates an oriented and orthochronous orthonormal frame $\left\{e_{\alpha}\right\}$ to every oriented eigenframe $\left\{\mathcal{U}_{i}\right\}$ : they are the 24 canonical frames of the Weyl tensor $[4,21]$. Then lemma 1 and proposition 1 offer us a covariant algorithm to determine these canonical frames.

Corollary 1. The Weyl canonical frames $\left\{e_{\alpha}\right\}$ of a Petrov type I spacetime may be determined to be

$$
e_{0}=\frac{-P_{0}(x)}{\sqrt{P_{0}(x, x)}} ; \quad e_{i}=U_{i}\left(e_{0}\right)
$$

with

$$
P_{0} \equiv \frac{1}{2}\left(g-\sum_{i=1}^{3} U_{i} \times U_{i}\right), \quad U_{i}=\operatorname{Re}\left(\sqrt{2} \mathcal{U}_{i}\right)
$$

and $\mathcal{U}_{i}$, the principal 2 -forms given in proposition 1 , and where $x$ is an arbitrary futurepointing vector.

Although there are 24 canonical frames, they define four unique orthogonal directions: one timelike principal direction and three spacelike principal directions.

\section{Principal 2-forms and Debever directions}

The 'light cones' defined by the metric $\mathcal{G}$ and the self-dual Weyl tensor $\mathcal{W}$ cut, generically, on four null directions of the self-dual 2-form space. Each one defines a null direction on the spacetime, usually called Weyl principal null direction or Debever direction [9]. These characteristic directions may be determined by solving a fourth degree algebraic equation with coefficients given by the components of $\mathcal{W}$ in a complex null frame. Then, an alternative approach to the Weyl algebraic classification follows analysing the multiplicity of the roots of this equation [9]. Type I appears as the case where four simple Debever directions exist. Depending on their multiplicity, these vectors satisfy an equation of the Sachs [10] hierarchy; for a simple Debever vector $l$ this equation can be written as:

$$
(l \wedge \mathcal{W}(l ; l) \wedge l)_{\alpha \beta \gamma \delta} \equiv-l_{[\alpha} \mathcal{W}_{\beta] \lambda \mu[\gamma} l_{\delta]} l^{\lambda} l^{\mu}=0
$$


A multiple Debever direction is always a principal direction of a Weyl principal 2-form [4], but a simple one has a more complicated relationship with principal 2-forms or with canonical frames. In Petrov type I, explicit expressions giving Debever directions in terms of a Weyl canonical frame $\left\{e_{\alpha}\right\}$ have been obtained in particular cases [12, 13, 14]. Here we generalize these results, presenting general expressions of the Debever directions in terms of principal 2-forms, so that, after results in the above section, a covariant algorithm to obtain Debever directions follows.

Every null vector $l$ satisfies $l \wedge \mathcal{G}(l ; l) \wedge l=0$. Thus, Sachs equation (10) implies that the simple Debever vectors may be characterized by the condition

$$
l \wedge \mathcal{A}_{i}(l ; l) \wedge l=0
$$

where, for every eigenvalue $\alpha_{i}, \mathcal{A}_{i} \equiv \mathcal{W}-\alpha_{i} \mathcal{G}$. Let us consider, for example, $i=3$. Then canonical form (7) implies

$$
\mathcal{A}_{3}=\sum_{A \neq 3}\left(\alpha_{3}-\alpha_{A}\right) \mathcal{U}_{A} \otimes \mathcal{U}_{A}
$$

So, $\mathcal{A}_{3}$ is a second-order tensor on the orthogonal to $\mathcal{U}_{3}$ subspace. In this subspace, two unique directions exist such that their bisectors are the principal 2-forms $\mathcal{U}_{A}$ and they form an angle $2 \Omega$, where

$$
\cos ^{2} \Omega=\frac{\alpha_{3}-\alpha_{1}}{\alpha_{2}-\alpha_{1}} .
$$

This complex invariant scalar coincides with the cross-ratio considered by Penrose [7]. If $\mathcal{V}_{\epsilon}(\epsilon= \pm 1)$ are the unitary 2 -forms in these characteristic directions, we have

$$
\mathcal{A}_{3}=\frac{\alpha_{2}-\alpha_{1}}{2} \mathcal{V}_{+} \tilde{\otimes} \mathcal{V}_{-}
$$

Thus, from (3), (11) and (12), it follows that Debever directions are the principal directions of the self-dual 2-forms $\mathcal{V}_{\epsilon}$. Then, from (2), and taking into account that the angle between $\mathcal{U}_{1}$ and $\mathcal{V}_{\epsilon}$ is $\Omega$, we can state

Proposition 2. Let $\alpha_{i}$ be the Weyl eigenvalues of a Petrov type I spacetime and let $\mathcal{U}_{i}$ be the principal 2-forms determined in proposition 1. Debever directions may be obtained as

$$
l_{\epsilon \pm} \propto\left[V_{\epsilon} \times V_{\epsilon} \underline{V_{\epsilon}}\right](x)
$$

with $V_{\epsilon}=\operatorname{Re}\left(\sqrt{2} \mathcal{V}_{\epsilon}\right)$ and

$$
\mathcal{V}_{\epsilon}=\cos \Omega \mathcal{U}_{1}+\epsilon \sin \Omega \mathcal{U}_{2}, \quad \epsilon= \pm 1 ; \quad \cos ^{2} \Omega=\frac{\alpha_{3}-\alpha_{1}}{\alpha_{2}-\alpha_{1}}
$$

and where $x$ is an arbitrary timelike vector.

Proposition 2 gives Debever directions from principal 2-forms and, after proposition 1, provides a covariant algorithm to obtain them. On the other hand, writing $U_{i}=e_{0} \wedge e_{i}$ and taking $x=e_{0}$ in expressions (13) and (14), a straightforward calculation gives us Debever directions in terms of canonical frames.

Corollary 2. Debever vectors of a Petrov type I spacetime can be written as

$$
l_{\epsilon \pm}=\cosh \psi e_{0} \pm \cos \phi e_{1} \pm \epsilon \sin \phi e_{2}+\epsilon \sinh \psi e_{3}, \quad \epsilon= \pm 1
$$

with

$$
\cos ^{2}(\phi-i \psi)=\frac{\alpha_{3}-\alpha_{1}}{\alpha_{2}-\alpha_{1}},
$$

where $\left\{e_{\alpha}\right\}$ is a Weyl canonical frame. 
It is easy to show the consistency between our expressions (15) and the results obtained by Debever in his early papers [6,9].

Notice that in the above study we have taken $i=3$ but we could equally have opted for any other eigenvalue. For each selection a different pair of unitary 2-forms like (14) exists, with Debever directions as principal directions: we call them Debever 2-forms. Thus, there are six Debever 2-forms, in accordance with all the possible pairs that may be considered with the four Debever directions of a type I spacetime.

Now let us pose the inverse problem: the determination of principal 2-forms and canonical frames using Debever vectors. Bonanos has given a geometric interpretation of the relationship between Debever vectors and canonical frames [15]. Here we present an alternative approach using our results, and offer a simple algorithm to calculate canonical frames in terms of Debever vectors.

Let $l_{\epsilon \pm}$ be the four Debever vectors normalized in such a way that $\left(l_{\epsilon+}, l_{\epsilon-}\right)=-2$, and consider the Debever 2-forms $\mathcal{V}_{\epsilon}=\frac{1}{\sqrt{2}}\left(l_{\epsilon-} \wedge l_{\epsilon+}-\mathrm{i} *\left(l_{\epsilon-} \wedge l_{\epsilon+}\right)\right)$. Then, taking into account proposition 2, the bisectors of $\mathcal{V}_{\epsilon}$ are eigendirections of the Weyl tensor. Therefore, it follows

Proposition 3. Let $\mathcal{V}_{\epsilon}=\frac{1}{\sqrt{2}}\left(l_{\epsilon-} \wedge l_{\epsilon+}-\mathrm{i} *\left(l_{\epsilon-} \wedge l_{\epsilon+}\right)\right)$ be two Debever 2-forms of a Petrov type I spacetime. Principal 2-forms $\left\{\mathcal{U}_{i}\right\}$ may be calculated as

$$
\begin{aligned}
& \mathcal{U}_{1}=\frac{1}{\sqrt{2}} \frac{\mathcal{V}_{+}+\mathcal{V}_{-}}{\sqrt{1-\left(\mathcal{V}_{+}, \mathcal{V}_{-}\right)}}, \\
& \mathcal{U}_{2}=\frac{1}{\sqrt{2}} \frac{\mathcal{V}_{+}-\mathcal{V}_{-}}{\sqrt{1+\left(\mathcal{V}_{+}, \mathcal{V}_{-}\right)}}, \\
& \mathcal{U}_{3}=\mathrm{i} \sqrt{2} \mathcal{U}_{1} \times \mathcal{U}_{2}
\end{aligned}
$$

Once the principal 2-forms have been determined by (16), we can use (9) to calculate the canonical frames. However, a more straightforward calculation follows reversing expressions (15). To sum up, we can state

Corollary 3. Let $k_{a}, a=1,2,3,4$, be the Debever vectors of a Petrov type I spacetime. Let us normalize them as follows:

$$
\begin{array}{ll}
l_{1}=\sqrt{\frac{\kappa_{42} \kappa_{43}}{\kappa_{12} \kappa_{13}}} k_{1}, & l_{2}=\sqrt{\frac{\kappa_{41} \kappa_{43}}{\kappa_{21} \kappa_{23}}} k_{2}, \\
l_{3}=\sqrt{\frac{\kappa_{41} \kappa_{42}}{\kappa_{31} \kappa_{32}}} k_{3}, & l_{4}=k_{4}
\end{array}
$$

where $\kappa_{a b}=\left(k_{a}, k_{b}\right)$. Then, the timelike and the spacelike principal directions may be obtained as

$$
e_{0} \propto \sum_{a=1}^{4} l_{a}, \quad e_{i} \propto l_{4}-\sum_{a=1}^{3}(-1)^{\delta_{i a}} l_{a}
$$

Finally, from our above analysis we will obtain the known results for type I degenerate cases (see [14] and references therein). The four Debever vectors span a three-dimensional space if, and only if, the real part of the Debever 2-forms (14) satisfy $V_{+} \wedge V_{-}=0$, i.e. when $\left(\mathcal{V}_{+}, \mathcal{V}_{-}\right)=\cos 2 \Omega$ is real. So, (14) implies that the ratio between every two eigenvalues is real or, equivalently, the scalar invariant $M=\frac{a^{3}}{b^{2}}-6$ is a positive real or infinity $\left(I\left(M^{+}\right)\right.$ or $I\left(M^{\infty}\right)$ in the Arianrhod et al classification [22]). In this case the eigenvalues may be strictly ordered by their modulus, and $\Omega$ is real $(\psi=0)$ if we take $\alpha_{3}$ as the shortest 
eigenvalue. As a consequence, (15) implies that the orthogonal to $e_{3}$ subspace contains the four Debever directions. Therefore, we can conclude

Corollary 4. In a Petrov type I spacetime, the four Debever vectors span a three-dimensional space iff $M=\frac{a^{3}}{b^{2}}-6$ is a positive real or infinity. This space is the orthogonal subspace to the spacelike principal direction associated with the shortest eigenvalue.

Thus we have recovered some previous results on this subject [14, 23]. Moreover, the known expressions for the Debever vectors in the degenerate type I cases [12, 13, 14] may also be easily deduced by taking $\psi=0$ in (15).

\section{Type I spacetimes admitting isotropic radiation}

The spacetimes admitting isotropic radiation with respect to a vorticity-free observer have been studied elsewhere [24], and their value for modelling the present universe has also been widely pointed out. These spacetimes may be characterized [25] as those admitting a timelike hypersurface-orthogonal conformal Killing vector or, in terms of the unitary vector field $u$, by conditions:

$$
\omega=0, \quad \sigma=0, \quad d\left(\dot{u}-\frac{1}{3} \theta u\right)=0
$$

$\omega, \sigma, \theta$ and $\dot{u}$ being, respectively, the rotation vector, the shear, the expansion and the acceleration of $u$. Therefore, the two first conditions (19) state that $u$ defines a shear-free and vorticity-free congruence, and so the Weyl tensor has real eigenvalues and is of Petrov type $\mathrm{I}, \mathrm{D}$ or 0 [12]. In type $\mathrm{I}, u$ is the unitary vector in the timelike principal direction, which will be called the Weyl principal observer. We summarize some of these known results $[25,12]$ in a lemma.

Lemma 2. In a Petrov type I spacetime, one has the following three equivalent statements.

(i) A hypersurface-orthogonal timelike conformal Killing vector exists.

(ii) A solution of Liouville equation, isotropic for a vorticity-free observer, exists.

(iii) The Weyl principal observer satisfies conditions (19).

In these spacetimes the Weyl principal observer keeps some of the main properties of the Robertson-Walker cosmological observer, in particular, that of observing isotropic radiation. Consequently it could play a similar role in building and interpreting type I cosmological models. Even, in generic type I universes, we can consider a privileged cosmological observer: the Weyl principal one. Thus, the interest in determining the timelike principal direction of a Petrov type I spacetime is plainly evident, a question that we have solved in corollary 1 .

Let us go back to the spacetimes considered in lemma 2. The three statements characterize them, but only the third gives us an invariant characterization because it asserts conditions on an invariant direction of the Weyl tensor. But this characterization is only useful after using our corollary 1 in order to calculate the timelike principal direction in terms of the Weyl tensor, and so obtain an intrinsic characterization of these spacetimes.

Indeed, conditions (19) may be easily written as equations on the projector tensor $h=u \otimes u+g$ :

$$
\begin{aligned}
& \omega=0, \quad \sigma=0 \Longleftrightarrow h_{\alpha}^{\lambda} h_{\beta}^{\mu} \nabla_{\lambda} h_{\mu}^{\nu}=h_{\alpha \beta} h^{\lambda \mu} \nabla_{\lambda} h_{\mu}^{\nu} \\
& d\left(\dot{u}-\frac{1}{3} \theta u\right)=0 \Longleftrightarrow d[4 h(\delta h)-\delta h]=0
\end{aligned}
$$


$\delta h$ being $(\delta h)_{\alpha}=-\nabla_{\lambda} h_{\alpha}^{\lambda}$. On the other hand, taking into account our above results, we can write $h$ in terms of principal 2-forms by using (5) and, after proposition 1, we obtain an expression of $h$ involving exclusively the metric tensor, Weyl concomitants and arbitrary directions: $h=h(W)$. Then we can conclude

Proposition 4. A Petrov type I spacetime admits an hypersurface-orthogonal timelike conformal Killing vector if, and only if, the Weyl tensor satisfies

$$
h_{\alpha}^{\lambda} h_{\beta}^{\mu} \nabla_{\lambda} h_{\mu}^{\nu}=h_{\alpha \beta} h^{\lambda \mu} \nabla_{\lambda} h_{\mu}^{\nu}, \quad d[4 h(\delta h)-\delta h]=0
$$

with $h=h(W) \equiv \frac{1}{2}\left[3 g-\sum_{i=1}^{3} U_{i} \times U_{i}\right], U_{i}=U_{i}(W) \equiv \operatorname{Re}\left(\sqrt{2} \mathcal{U}_{i}\right), \mathcal{U}_{i}$ being the principal 2 -forms determined in proposition 1 . Then, the conformal Killing direction is

$$
u \propto(h-g)(x)
$$

where $x$ is an arbitrary future-pointing vector.

This proposition affords an intrinsic characterization, through equations involving exclusively Weyl concomitants, of the type I universes admitting a solution of the Liouville equation isotropic for a vorticity-free cosmological observer. A wider family of spacetimes, where there exist solutions of the Boltzmann equation which are isotropic for a vorticityfree observer [26], may be useful in modelling the pre-recombination epoch [27]. In these spacetimes an umbilical synchronization exists [24] and, in the type I case, they may be characterized as those where the Weyl tensor satisfies the first equation in (22).

\section{Acknowledgments}

We would like to thank $\mathbf{J}$ A Morales and B Coll for their encouragement and comments. This work has been supported by the Generalitat Valenciana, project GV 2207/94.

\section{References}

[1] Petrov A Z 1954 Sci. Not. Kazan Univ. 11455

[2] Géhéniau J 1957 Comptes Rendus Acad. Sci. 244723

[3] Bel L 1959 La radiation gravitationelle in Les théories relativistes de la gravitation (Royaumont)

[4] Bel L 1962 Cah. de Phys. 1659

[5] Ruse H S 1936 Proc. London Math. Soc. 41302

[6] Debever R 1964 Cah. de Phys. 168-169 303

[7] Penrose R 1960 Ann. Phys. 10171

[8] Debever R 1956 Bull. Acad. R. Belgique 42313

[9] Debever R 1959 Bull. Soc. Math. Belgique 10112

[10] Sachs R 1961 Proc. R. Soc. A 264302

[11] d'Inverno R and Russell-Clark R 1971 J. Math. Phys. 121258

[12] Trümper M 1965 J. Math. Phys. 6584

[13] Narain U 1970 Phys. Rev. D 2278

[14] McIntosh C B G, Arianrhod R, Wade S T and Hoenselaers C 1994 Class. Quantum Grav. 111555

[15] Bonanos S 1989 Gen. Rel. Grav. 21953

[16] Bona C, Coll B and Morales J A 1992 J. Math. Phys 33670

[17] Coll B and Ferrando J J 1989 J. Math. Phys 302918

[18] Ferrando J J and Sáez J A 1995 On the principal directions of the Weyl tensor in Inhomogeneous Cosmological Models, Proc. 'Relativity Meeting 1994' (Singapore: World Scientific)

[19] Ferrando J J, Morales J A and Sáez J A 1996 in preparation

[20] Coll B and Ferrando J J 1988 Gen. Rel. Grav. 2051

[21] Bonanos S 1989 Gen. Rel. Grav. 211073

[22] Arianrhod R and McIntosh C B G 1992 Class. Quantum Grav. 91969

[23] McIntosh C B G and Arianrhod R 1990 Class. Quantum Grav. 7 L213 
[24] Ferrando J J, Morales J A and Portilla M 1992 Phys. Rev D 46578

[25] Ehlers J, Geren P and Sachs J 1968 J. Math. Phys. 91344

[26] Treciokas R and Ellis G F R 1971 Commun. Math. Phys. 231

[27] Ferrando J J, Morales J A and Portilla M in preparation 